\title{
Höhere Adenomdetektionsrate unter Wasser
}

Wird bei der Koloskopie Wasser statt Luft insuffliert, erhöht sich die Adenomdetektionsrate (ADR) signifikant. Gleichzeitig wird die Untersuchung für den Patienten besser verträglich, dauert allerdings geringfügig länger.

Hintergrund und Fragestellung: Die ADR gilt als wichtiger Qualitätsparameter für die Screeningkoloskopie. Anhand von Screeningstudien aus westlichen Ländern wird eine ADR von $\geq 30 \%$ bei Männern und $\geq 20 \%$ bei Frauen gefordert. Sinkt sie unter $20 \%$, ist mit einem Anstieg von Intervallkarzinomen $\mathrm{zu}$ rechnen - eine Katastrophe für den Patienten und ein Menetekel für den Endoskopiker. In Studien aus China liegt die ADR nur bei $12-19 \%$, was möglicherweise durch einen geringeren technischen Standard der Endoskope bedingt ist. Nun wurde untersucht, ob dieser Wert durch eine einfache technische Modifikation der Untersuchung verbessert werden kann.

Patienten und Methoden: In einer prospektiven, randomisierten, kontrollierten Studie an sechs chinesischen Zentren wurden Screening-, Überwachungs- und diagnostische Koloskopien mittels Wasserinsufflation (WI) oder konventionell mittels Luftinsufflation (LI) durchgeführt. Die Vorbereitung erfolgte mittels 4 Liter PEG-Lösung in aufge-

$\mathrm{H}$ Jia et al. Water exchange method significantly improves adenoma detection rate: a multicenter, randomized controlled trial. Am J Gastroenterol 2017; 112: $568-76$ teilter Dosis. Primärer Endpunkt war die ADR, doch wurden auch andere Qualitätsparameter wie die Rate der Zökumintubation, die Dauer der Untersuchung und die Patientenzufriedenheit erfasst. Die Untersuchungen erfolgten durch Endoskopiker mit Erfahrung von mindestens 1.000 LI- und 50 WI-Untersuchungen. An Geräten kamen die Videokoloskope EC-590WM von Fujinon und CF-Q260 von Olympus zum Einsatz. Alle Untersuchungen erfolgten mittels Weißlicht und $37^{\circ} \mathrm{C}$ warmem Wasser. Hilfsmittel wie Färbungen, Narrow Band Imaging, elektronische Farbverstärkung oder Autofluoreszenz wurden nicht eingesetzt.

Ergebnisse: Von April 2014 bis Juli 2015 wurden 3.303 Patienten (18 bis 80 Jahre) untersucht, 1.653 mittels WI, 1.650 mittels LI. Die Basisdaten der Patientengruppen waren vergleichbar. Die Gesamt-ADR in der WI-Gruppe betrug 18,3\%, in der LIGruppe 13,4 \% (RR 1,45, 95\%-Konfidenzintervall [KI] 1,20 - 1,75; $\mathrm{p}<0,001)$. Bei den Screeningpatienten über 50 Jahre lag die ADR nach WI bei 29,4\%, nach LI bei 22,9\% (RR 1,09, 95\%-KI $1,00-1,19 ; p=0,040)$. Die ADR fortgeschrittener und sessiler serratierter Adenome betrug in den Gruppen 6,2\% respektive $4,5 \%$, was einem erhöhten relativen Risiko von $16 \%$ in der LIGruppe entspricht. Unter WI fanden sich auch mehr kleine $(<10$ $\mathrm{mm}$ ), flache und tubuläre Adenome. Dies mag für die Prognose wenig relevant sein, wirft aber ein Licht auf die grundsätzlich besseren Detektionsmöglichkeiten von Adenomen insgesamt.

Im Vergleich zur LI- war die Zeit bis zum Erreichen des Zökums in der WI-Gruppe mit 7,4 versus 4,9 Minuten signifikant länger, die Rückzugszeit dagegen eine Minute geringer. Patienten, die keine Sedierung bevorzugten, berichteten über signifikant geringere Schmerzen während der Untersuchung. Dementsprechend wollten 94,5\% der nicht sedierten Patienten in der WI-Gruppe die Untersuchung erneut in der gleichen Weise durchführen lassen, in der LI-Gruppe waren es $91,5 \%$.

\section{- Kommentar von Hermann S. Füeßl, München}

\section{Einfache Maßnahme führt zu besseren Ergebnissen}

Obwohl auch in den westlichen Ländern seit etwa 20 Jahren bekannt ist, dass eine Insufflation von Wasser anstelle von Luft die Ergebnisse der Koloskopie verbessern kann, hat sich die Wasserkoloskopie in der Praxis nicht durchgesetzt. Die wesentlichen Gründe dafür liegen wahrscheinlich in der längeren Untersuchungsdauer durch ständiges Spülen und Absaugen. Bedenkt man aber, dass viele der mit Luftinsufflation durchgeführten Untersuchungen wegen Stuhlverunreinigung nicht optimal beurteilbar sind, so verwundert dies doch. In der vorliegenden Studie aus China schwingt einiger Stolz mit, dass die bislang nicht konkurrenzfähige ADR mit dieser einfachen Maßnahme ohne Investitionen in die Geräteausstattung dem westlichen Standard angenähert werden konnte. Hierzulande bevorzugen wir technologische (und entsprechend kostspielige) Lösungen, zum Beispiel mit NBI oder dem Rundblickendoskop, wobei beide Verfahren eine ungenügende Darmreinigung nicht kompensieren können. Die Schmerzreduktion bei der Wasserkoloskopie spielt bei uns auch nicht die große
Rolle, weil die meisten Patienten eine kontinuierliche Sedierung bekommen, meistens sogar durch einen Anästhesisten. Der Studie kommt das Verdienst zu, gezeigt zu haben, wie auch in medizinisch weniger entwickelten Ländern mit einer einfachen Modifikation der Untersuchung sehr gute Ergebnisse erzielt werden können.

In den letzten Jahren wird zunehmend anstelle von Luft $\mathrm{CO}_{2}$ zur Insufflation verwendet, um den Komfort bei nicht sedierten Patienten zu verbessern. Hierzu liegt auch eine erste kontrollierte Studie vor, die die Wasserkoloskopie mit der $\mathrm{CO}_{2}$-Insufflation verglichen hat. Die Häufigkeit von starken und mittelstarken Schmerzen war bei beiden Verfahren gleich, lediglich in der Kategorie der leichten Schmerzen wies die Wasserkoloskopie Vorteile auf.

Fazit: Die Wasserkoloskopie weist hinsichtlich der ADR und des Patientenkomforts durchaus Vorteile auf. Wahrscheinlich ist diese Methode unter dem Druck des Zeitdiktats in Vergessenheit geraten. 